\title{
New Drugs on the Block-Emerging Treatments for Nonalcoholic Steatohepatitis
}

\author{
Sanja Stojsavljevic-Shapeski ${ }^{1}$, Marko Duvnjak ${ }^{* 2,3}$, Lucija Virovic-Jukic ${ }^{1,4}$, \\ Davor Hrabar ${ }^{1,4}$ and Lea Smircic Duvnjak ${ }^{4,5}$
}

\begin{abstract}
${ }^{1}$ Department of Gastroenterology and Hepatology, Clinical Hospital Center Sestre Milosrdnice, Zagreb, Croatia; ${ }^{2}$ Polyclinic Duvnjak, Zagreb, Croatia; ${ }^{3}$ University of Applied Health Science, Zagreb, Croatia; ${ }^{4}$ School of Medicine, University of Zagreb, Zagreb, Croatia; ${ }^{5}$ Vuk Vrhovac University Clinic-UH Merkur, Zagreb, Croatia
\end{abstract}

\begin{abstract}
Patients with nonalcoholic steatohepatitis (NASH) are at higher risk of progression to advanced stages of fibrosis, cirrhosis, hepatocellular carcinoma and other end-stage liver disease complications. When addressing treatment of $\mathrm{NASH}$, we have limited approved options, and the mainstay of therapy is lifestyle intervention. Extensive research and revelation in the field of pathogenesis of NASH has offered new possibilities of treatment and emerging new drugs that are being tested currently in numerous preclinical and clinical trials. These drugs target almost all steps in the pathogenesis of NASH to improve insulin sensitivity, glucose and lipid metabolism, to inhibit de novo lipogenesis and delivery of lipids to the liver, and to influence apoptosis, inflammation and fibrogenesis. Although NASH is a multifactorial disease, in the future we could identify the predominating pathological mechanism and, by choosing the most appropriate specific medication, tailor the treatment for every patient individually.
\end{abstract}

Citation of this article: Stojsavljevic-Shapeski S, Duvnjak $M$, Virovic-Jukic L, Hrabar D, Smircic Duvnjak L. New drugs on the block-Emerging treatments for nonalcoholic steatohepatitis. J Clin Transl Hepatol 2020;000(000):000-000. doi: $10.14218 /$ JCTH.2020.00057.

\section{Introduction}

Nonalcoholic steatohepatitis (NASH) and nonalcoholic fatty liver are histologically two distinguishable subtypes of nonalcoholic fatty liver disease (NAFLD). Nonalcoholic fatty liver requires more than $5 \%$ fat infiltration of the liver and $\mathrm{NASH}$, alongside fat infiltration, is characterized by inflammation

Keywords: Fatty liver; Steatohepatitis; Nonalcoholic fatty liver disease; Treatment.

Abbreviations: ALT, alanine aminotransferase; ASK-1, apoptosis signal-regulating kinase 1 ; BMI, body mass index; CCR, C-C chemokine receptor type; DPP4, dipeptidyl peptidase 4; FGF, fibroblast growth factor; FXR, farnesoid $X$ receptor; GLP-1, glucagon-like peptide-1; MAFLD, metabolic-associated fatty liver disease; $M R$, magnetic resonance; NAFLD, nonalcoholic fatty liver disease; NAS, NAFLD activity score; NASH, nonalcoholic steatohepatitis; NFKB, nuclear factor kappa B; PPAR, peroxisome proliferator-activated receptor; SGLT, sodium-glucose co-transporter.

Received: 20 June 2020; Revised: 18 September 2020; Accepted: 21 October 2020

*Correspondence to: Marko Duvnjak, Polyclinic Duvnjak, Kukuljeviceva 2, Zagreb 10000, Croatia. Tel: +38-5989838930, E-mail: marko.duvnjak1@gmail. and hepatocyte injury. ${ }^{1}$ Although hepatic steatosis within NAFLD is a widespread disease, with prevalence in some parts of the world up to $40 \%, 2 \mathrm{NASH}$ is present in only $10 \%$ to $20 \%$ of individuals with NAFLD, but when accompanied by significant fibrosis is associated with increased overall mortality, primarily from cardiovascular diseases. ${ }^{1}$ Population projection models estimate that $3 \%$ to $6 \%$ of adults have NASH, and according to current trends, the prevalence of NASH is expected to rise by $15 \%$ to $56 \%$ until $2030.3,4$

Patients with NASH have a higher risk of progression to advanced stages of fibrosis, cirrhosis, hepatocellular carcinoma and other end-stage liver disease complications. ${ }^{5}$ At the time of NASH diagnosis about $25 \%$ of patients have a moderate to severe stage of fibrosis $(F>2)$, and in around $40 \%$ of NASH patients fibrosis will progress at a rate of 1 stage per 10 years. ${ }^{6}$ Although often clinically silent, more that $20 \%$ of patients with NASH will develop end-stage liver disease over their lifetime.7,8 A meta-analysis of 86 studies and more than 8 million patients from 22 countries has shown that in comparison with NAFLD, NASH has greater overall mortality ( 11.77 to 0.77 per 1,000 person-years) and liver related mortality (25.56 to 15.44 per 1,000 person-years). ${ }^{6}$

When addressing treatment of $\mathrm{NASH}$, we have limited approved options, and the mainstay of therapy is lifestyle intervention, including changes in diet and exercise regimes, with an emphasis on weight reduction of more than $7 \%, 1,9,10$

Several drugs have been proposed for treating NASH, but according to the guidelines of European and American societies, metformin (an insulin sensitizer) is not recommended because it showed no effect on liver histology although it has a beneficial effect on insulin resistance and alanine aminotransferase (ALT) levels.1,11 Pioglitazone, a thiazolidinedione and intra-nuclear peroxisome proliferator-activated receptor (PPAR) y agonist that is in use for diabetes mellitus treatment, was evaluated in several trials for the treatment of NASH. In the PIVENS randomized controlled trial, patients with biopsy-proven NASH without diabetes mellitus received vitamin E (800 IU/day) and pioglitazone $(30 \mathrm{mg} /$ day) for 96 weeks and were compared with patients who received placebo. ${ }^{12}$ The therapy with vitamin E was associated with amelioration of NASH (43\% vs. 19\%); however, when compared with the placebo, the pioglitazone therapy was not that successful ( $34 \%$ and $19 \%$ ). ${ }^{12}$ Both therapies were associated with reduction of hepatic steatosis, inflammation and liver laboratory tests but without an improvement in fibrosis scores. ${ }^{12}$ Pioglitazone is, however, associated with substantial side-effects, such as weight gain, fluid retention, heart failure and bone loss, so the American Association for the Study of Liver Diseases 2018 Guidelines state that pioglitazone may be used in biopsy-proven NASH 
Table 1. New drugs in phase 2 and phase 3 clinical trials

\begin{tabular}{|c|c|c|c|c|c|}
\hline Agent & $\begin{array}{l}\text { Mechanism } \\
\text { of action }\end{array}$ & Treatment/intervention & Patients & Phase & $\begin{array}{l}\text { ClinicalTrials. } \\
\text { gov identifier }\end{array}$ \\
\hline Elafibranor & $\begin{array}{l}\text { Dual PPARa and } \\
\text { PPARס agonist }\end{array}$ & Elafibranor (120 mg) vs. placebo & $\begin{array}{l}\text { NASH (NAS >4) } \\
\text { and F2/F3 }\end{array}$ & 3 & NCT02704403 \\
\hline Saroglitazar & $\begin{array}{l}\text { Dual PPARa and } \\
\text { PPARY agonist }\end{array}$ & $\begin{array}{l}\text { Saroglitazar }(1,2 \text { or } 4 \\
\text { mg) vs. placebo }\end{array}$ & $\begin{array}{l}\text { NASH and/or NAFLD } \\
\text { (biopsy or noninvasive) }\end{array}$ & 2 & NCT03061721 \\
\hline Lanifibranor & pan-PPAR agonist & $\begin{array}{l}\text { Lanifibranor ( } 800 \mathrm{mg} \text { and } \\
1,200 \mathrm{mg} \text { ) vs. placebo }\end{array}$ & NASH (biopsy) & 2 & NCT03008070 \\
\hline Liraglutide & $\begin{array}{l}\text { GLP-1 receptor } \\
\text { agonist }\end{array}$ & $\begin{array}{l}\text { Liraglutide }(0,6-3 \mathrm{mg}) \text { vs. exercise } \\
+ \text { diet vs. bariatric surgery }\end{array}$ & $\begin{array}{l}\text { NASH (biopsy or } \\
\text { noninvasive) }\end{array}$ & 3 & NCT02654665 \\
\hline Semagultide & $\begin{array}{l}\text { GLP-1 receptor } \\
\text { agonist }\end{array}$ & $\begin{array}{l}\text { Semagultide }(0.1,0.2 \\
0.4 \mathrm{mg}) \text { vs. placebo }\end{array}$ & NASH (biopsy) & 2 & NCT02970942 \\
\hline Dapagliflozin & SGLT inhibitor & Dapagliflozin $(10 \mathrm{mg})$ vs. placebo & NASH and DM (biopsy) & 3 & NCT03723252 \\
\hline Pegbelfermin & FGF21 analog & Pegbelfermin (3 doses) vs. placebo & NASH and F3 (biopsy) & 2 & NCT03486899 \\
\hline Cenicriviroc & $\begin{array}{l}\text { CCR2/CCR5 } \\
\text { antagonist }\end{array}$ & Cenicriviroc (150 mg) vs. placebo & $\begin{array}{l}\text { NASH and F2/ } \\
\text { F3 (biopsy) }\end{array}$ & 3 & NCT03028740 \\
\hline Tropifexor & FXR agonist & $\begin{array}{l}\text { Tropifexor monotherapy vs. } \\
\text { combination with cenicriviroc }\end{array}$ & $\begin{array}{l}\text { NASH with F2/ } \\
\text { F3 (biopsy) }\end{array}$ & 2 & NCT03517540 \\
\hline Resmetirom & $\begin{array}{l}\text { Thyroid hormone } \\
\text { receptor } \beta \text { agonist }\end{array}$ & $\begin{array}{l}\text { Resmetirom ( } 80 \mathrm{mg}, \\
100 \mathrm{mg}) \text { vs. placebo }\end{array}$ & NASH (NAS>4) & 3 & NCT03900429 \\
\hline Firsocostat & $\begin{array}{l}\text { Acetyl-CoA } \\
\text { carboxylase inhibitor }\end{array}$ & $\begin{array}{l}\text { Firsocostat vs. fenofibrate, } \\
\text { cilofexor, selonsertib }\end{array}$ & $\begin{array}{l}\text { NASH (F2/F3 and } \\
\text { F4/cirrhosis) }\end{array}$ & 2 & $\begin{array}{l}\text { NCT02781584, } \\
\text { NCT03449446 }\end{array}$ \\
\hline
\end{tabular}

Abbreviations: CCR2/CCR5, C-C chemokine ligand types 2 and 5; DM, diabetes mellitus; FGF21, fibroblast growth factor-21; FXR, farnesoid X receptor; GLP-1, glucagon-like peptide-1; NAFLD, nonalcoholic fatty liver disease; NAS, NAFLD activity score; NASH, nonalcoholic steatohepatitis; PPAR, peroxisome proliferator-activated receptor; SGLT, sodium-glucose co-transporter.

patients after discussing the risks and benefits. ${ }^{1}$ Vitamin $\mathrm{E}$ has been associated with increased incidence of intracranial bleeding and prostate cancer; however, NASH patients who could have benefited from vitamin $\mathrm{E}$ were not included in the study nor did the study take into account other confounding factors (smoking, supplements). ${ }^{13}$ Orlistat, as well as lipid lowering agents used in treating hyperlipidemia, have been used in patients with $\mathrm{NASH}$, and although their use is safe, the outcomes of their effect on treating NASH are inconclusive. ${ }^{1,11}$

As a result of better understanding the underlying processes in the development of NAFLD and NASH, as well as a long withstanding opinion that the nomenclature for NAFLD and NASH doesn't suffice, an international working group represented by Eslam et al. ${ }^{14}$ proposed a change in nomenclature to metabolic-associated fatty liver disease or "MAFLD".14 This change would, in their opinion, better depict the character of this disease and patients that suffer from it, and alienate the labeling alcoholic burden. This could also propose a change in the study design of preclinical and clinical trials that would take into consideration the specific heterogeneity of the pathological pathways and specific characteristics of the patients and thus give more reliable results for the studied drugs. ${ }^{14}$

Extensive research and revelation in the field of pathogenesis of NASH has offered new possibilities of treatment and emerging new drugs that are being tested now in numerous preclinical and clinical trials (Table 1 ). These drugs target almost all steps in the pathogenesis of NAFLD and $\mathrm{NASH}$, aiming to improve insulin resistance, glucose and lipid metabolism, to inhibit lipogenesis and delivery of lipids to the liver, and to influence apoptosis, inflammation and fibrogenesis. ${ }^{15}$ Figure 1 depicts the proposed mechanisms of action of these agents in the pathogenesis of NAFLD and $\mathrm{NASH}$.

Intestinal microbiome and associated dysbiosis has emerged as an important factor that indirectly, through intestinal permeability and bacterial metabolites, influences several mechanisms responsible for NASH progression. The therapeutic options that could influence these steps are depicted in Figure 2.

\section{Drugs improving insulin sensitivity and modulating glucose and lipid metabolism}

\section{PPARs}

PPARs are nuclear receptors that regulate metabolic homeostasis, cell differentiation and immune-inflammation. ${ }^{16}$ We distinguish several PPAR intracellular receptor subtypes, as they exert different distribution and actions in different tissues. ${ }^{16}$ The first extensively studied PPARY agonist, pioglitazone, already mentioned in the introduction, modulates glucose uptake, insulin signaling, fatty acid uptake, triglyceride synthesis and hydrolysis, as well as inflammation and maturation of macrophages. ${ }^{16}$

PPARa is most extensively pronounced in the liver and exerts is regulatory roles through fatty acid transport and $\beta$-oxidation to deliver lipids in the liver, and has been shown to decrease triglycerides and increase high-density lipoprotein cholesterol in serum. ${ }^{17}$ It also influences inflammation through nuclear factor-kappa B (NFKB) action modulation and reduces the expression of acute-phase genes. ${ }^{17}$ PPARy is mostly expressed in the adipose tissue and controls lipogenesis, adipocyte differentiation, and glucose metabolism. ${ }^{18}$ By promoting the storage of fatty acids, such as triglycerides, PPARY acts as an insulin sensitizer and prevents ectopic fat accumulation. ${ }^{18,19}$ Maeda et al. ${ }^{20}$ showed that PPARY agonists caused a significant rise in plasma adiponectin concentrations. Adiponectin is a protein derived 
Stojsavljevic-Shapeski S. et al: Emerging treatments for NASH

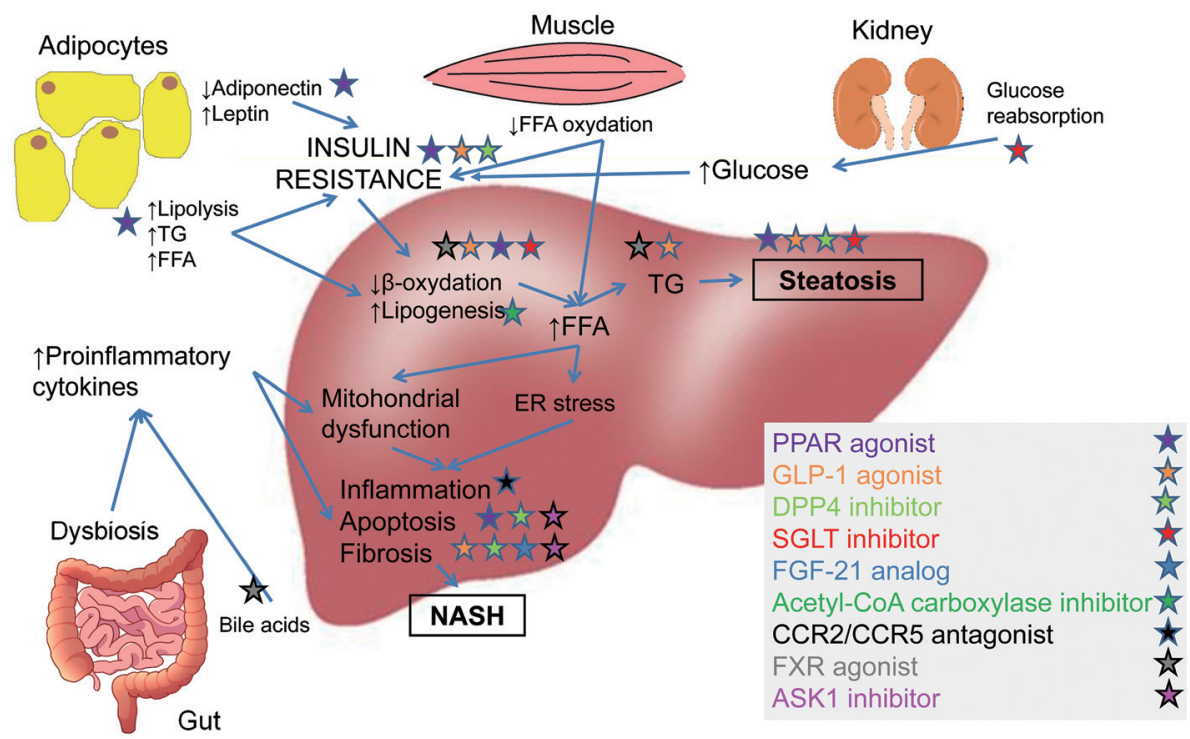

Fig. 1. Proposed mechanisms of action of new agents for treatment of NASH. Abbreviations: ASK-1, apoptosis signal-regulating kinase 1 ; CCR $2 / C C R 5, C-C$ chemokine ligand types 2 and 5; DPP4, dipeptidyl peptidase 4; ER, endoplasmic reticulum; FFA, free fatty acids; FGF21, fibroblast growth factor-21; FXR, farnesoid X receptor; GLP-1, glucagon-like peptide-1; NASH, nonalcoholic steatohepatitis; PPAR, peroxisome proliferator-activated receptor; SGLT, sodium-glucose co-transporter; TG, triglycerides.

from fat tissue that possesses anti-atherogenic properties and suppresses cytokine production from macrophages and expression of adhesion molecules in vascular endothelial cells. PPARס agonists produce similar effects as PPARa on liver lipid metabolism, exhibit a positive influence on insulin sensitivity, and promote an alternative activation effect on macrophages and Kupffer cells that leads to attenuation of tissue inflammation. 21,22

Elafibranor is a dual PPARa and PPARס agonist, that was investigated in a phase II multicenter, randomized placebo-controlled study at two dose regimes ( $80 \mathrm{mg}$ and 120 $\mathrm{mg}$ once a day versus placebo) over 52 weeks in biopsyproven NASH patients. ${ }^{23}$ Treatment with $120 \mathrm{mg}$ of elafibranor reduced liver enzymes, lipid values, glucose profiles, and markers of systemic inflammation in comparison with placebo, and there was a statistically significant amelioration of NASH activity without aggravation of fibrosis. ${ }^{23}$ Furthermore, the patients that experienced NASH regression had a significant decline in fibrosis stage compared to those without NASH regression. ${ }^{23}$ Elafibranor, although, was welltolerated and caused an increase in serum creatinine level that was reversible. ${ }^{23} \mathrm{~A}$ phase III multicenter study (RESOLVE-IT) on elafibranor (120 mg per day versus placebo) is ongoing and planned to enroll 2,000 patients with liver biopsy-proven NASH (NAFLD activity score (NAS) of $>4$ ) and F2-3 fibrosis (NCT02704403). ${ }^{24}$

Saroglitazar is a dual PPARa and PPARy agonist that exhibits a predominant PPARa effect with a moderate PPARY

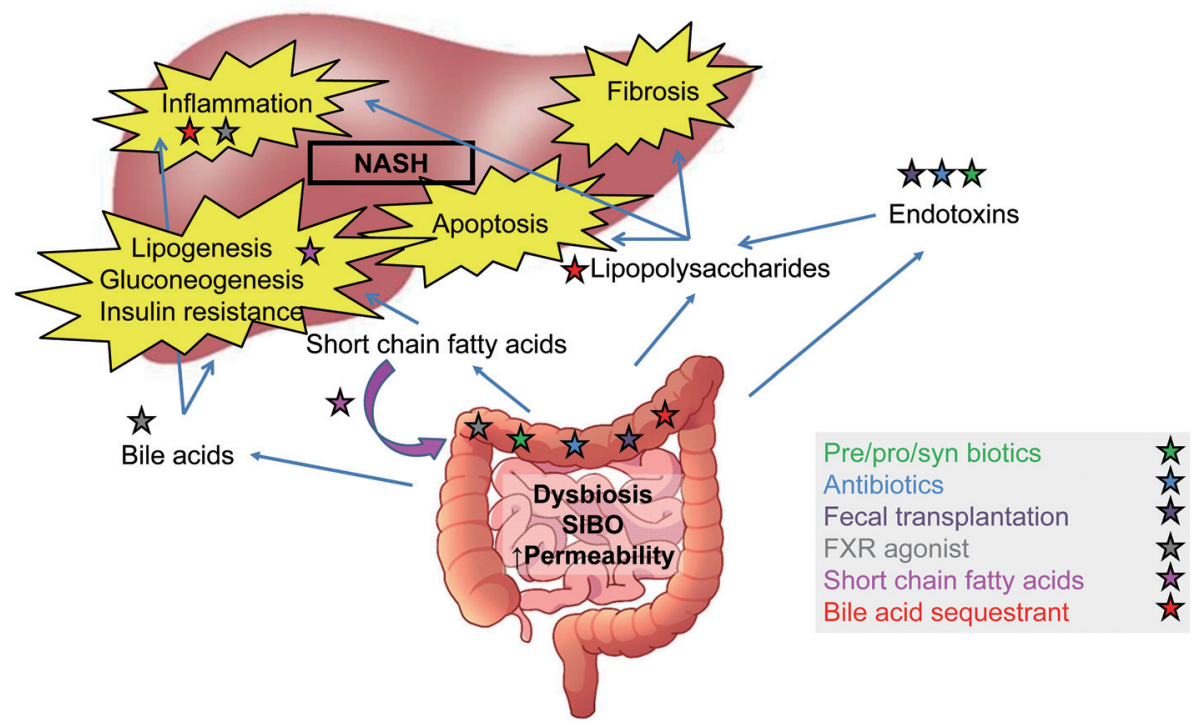

Fig. 2. Proposed mechanism of action of treatments for NASH targeting microbiome changes. Abbreviations: NASH, nonalcoholic steatohepatitis; FXR, farnesoid X receptor; SIBO, small intestinal bacterial overgrowth. 
effect; as such, it provides a positive effect on lipid metabolism and insulin sensitivity without the side effects caused by PPARY activation. ${ }^{25}$ Since it has a nonrenal route of elimination, it has been shown to be safe in patients with deteriorated renal function. ${ }^{25} \mathrm{~A}$ review of 18 selected studies on patients with diabetic dyslipidemia that were treated with saroglitazar $4 \mathrm{mg}$ once daily for at least 12 weeks showed a consistent mean regression in lipid levels and glycosylated hemoglobin levels with an increase in mean high-density lipoprotein cholesterol levels from baseline as well as an incline in ALT levels and fatty liver (evaluated by FibroScan ${ }^{\mathrm{TM}}$ ) in NAFLD patients with diabetic dyslipidemia. ${ }^{26}$ There is an ongoing phase II study on saroglitazar on NASH/NAFLD patients that will investigate the safety of treatment and impact on serum ALT levels (NCT03061721). ${ }^{27}$

Lanifibranor is a next-generation pan-PPAR agonist that in a NAFLD mouse model improved insulin resistance and steatohepatitis (biopsy-assessed hepatic steatosis, inflammation, ballooning and fibrosis), that combines and exceeds specific effects of the single PPAR agonists. ${ }^{18}$ Currently, efficacy and the safety of two doses (800 $\mathrm{mg}$ and 1,200 mg) of lanifibranor per day for 24 weeks is being evaluated in a phase II study versus placebo in adult NASH patients with moderate to severe necroinflammation without cirrhosis (NCT03008070).

\section{Glucagon-like peptide receptor agonists and dipepti- dyl peptidase 4 inhibitors}

Glucagon-like peptide-1 (GLP-1), recognized as physiologic incretin, is a hormone secreted from the distal ileum and coIon that increases insulin synthesis and secretion, decreases glucagon secretion, decreases hepatic gluconeogenesis, suppresses appetite, and delays gastric emptying. ${ }^{28}$ It has been shown that hepatocytes express GLP-1 receptors and that GLP-1 agonists reduce steatosis and influence lipid metabolism by decreasing lipogenesis and increasing oxidation of fatty acids. 29

Liraglutide, a first class GLP-1 receptor agonist, was studied on a hepatic stellate cell (rat and human) model, and it was found that liraglutide markedly improved the stellate cell phenotype and diminished cell proliferation. ${ }^{30}$ Rats with cirrhosis treated with liraglutide had lower portal pressure, lower intrahepatic vascular resistance, and significant improvement in fibrosis and endothelial function. ${ }^{30}$ These antifibrotic effects of liraglutide therapy were also recorded in human liver and the proposed mechanism is an GLP1-R-independent and NFKB-Sox9-dependent one. ${ }^{30}$ Furthermore, metabolic and hepatic beneficiary effects of liraglutide were studied in an obese NASH mouse model, and found reduced body weight, reduced hepatis steatosis, and reduced collagen $1 \mathrm{a} 1$ and galectin- 3 content. ${ }^{31}$ In a randomized, phase 2 study, liraglutide was compared to placebo in obese, biopsyconfirmed NASH patients. ${ }^{31}$ After the treatment with liraglutide, $40 \%$ of patients had NASH resolution compared with $9 \%$ in the placebo group. Only $9 \%$ of patients on liragultide versus $36 \%$ of patients on placebo had progression of fibrosis. ${ }^{32}$ Results also showed improvement in the metabolic risk factors such as weight, glucose and high-density lipoprotein cholesterol levels; the main reported adverse events were mild to moderate, and included diarrhea, constipation and loss of appetite. ${ }^{32}$ Currently ongoing is a phase 3 study that is comparing effects of liraglutide and bariatric surgery on anthropometric measures, liver function, insulin resistance, endothelial function and biomarkers of NASH (CGHLiNASH, NCT02654665). A recently published study on 30 obese NAFLD patients showed that liraglutide administered at $3 \mathrm{mg}$ daily for 26 weeks, followed by 26 weeks of only weight gain prevention, compared with diet and exercise modifications only, had significant reductions in weight, liver fat fraction (measured by magnetic resonance imaging), serum ALT and caspase-cleaved cytokeratin-18 at 26 weeks. ${ }^{33}$ However, those benefits were not sustained after discontinuation of treatment, in contrast with effects of lifestyle modification. ${ }^{33}$

Exenatide, also a GLP-1 receptor agonist, in a NASH mouse model showed an improvement of mitochondrial tricarboxylic acid cycle flux after a 8-week treatment, a significant decrease in insulin resistance, steatosis, hepatocyte lipotoxicity and hepatic triglyceride content as well as lower expression of hepatic lipogenic genes (Srebp1C, Cd36) and genes involved in inflammation and fibrosis. ${ }^{34}$ Exenatide treatment for 12 weeks ( $5 \mathrm{mg}$ twice daily for 4 weeks then $10 \mathrm{mg}$ twice daily for 8 weeks) combined with insulin glargine in diabetic, obese, NAFLD patients was associated with a greater reduction of body weight, waist circumference, liver fat (appraised by ultrasound) and liver enzymes than with intensive insulin therapy (93\% vs. $67 \%)$; at the end of treatment, up to $43 \%$ of patients had no liver steatosis. 35 The most common side effects were similar to liraglutide, and were found in up to $40 \%$ of patients. ${ }^{35} \mathrm{~A}$ relatively small open-label study on eight patients with diabetes mellitus and biopsy-proven NASH found that 28 weeks of exenatide treatment made no significant difference in liver histology, and only three of the eight subjects did meet the primary end point of improved histopathology, with 1 to 2 point fibrosis improvement seen in four subjects and fibrosis worsening by 1 point in one subject and staying the same in three subjects. ${ }^{36}$ More studies on exenatide are needed to draw firmer conclusions on its role in treating NASH patients.

Semaglutide, a novel GLP-1 receptor agonist, was investigated in a recently completed phase 2 placebo-controlled trial comparing the efficacy and safety of different doses in $320 \mathrm{NASH}$ patients and the results are awaited (NCT02970942). Since NASH patients have a greater risk of cardiovascular mortality, semaglutide could have potential benefit compared to other GLP-1 receptor agonists, since it was shown to be able to prevent cardiovascular events as well as reduce body mass and ALT level. ${ }^{37,38}$

Dipeptidyl peptidase 4 (DPP4) inhibitors exert their effect by blocking the enzyme DPP4, which is involved in the degradation of GLP-1 and other incretins. ${ }^{28}$ Serum DPP4 levels are elevated in NASH patients and correlate well with the histopathological severity of NASH. DPP4 levels are also positively associated with liver fibrosis and hepatocyte apopotosis. ${ }^{28}$ Sitagliptine, an DPP4 inhibitor, prevented infiltration of adipose tissue by $\operatorname{CD} 8(+)$ T-cells and M1 macrophages, decreased PAI-1 expression, and had a positive effect on liver lipid metabolism and liver fat infiltration. ${ }^{39}$ It was also shown in a mouse model that sitagliptine could prevent the progression of hepatocellular carcinoma related to NASH. ${ }^{40}$ However, a relatively small study on biopsy-proven NASH patients after 24 weeks of sitagliptine $(100 \mathrm{mg})$ showed no improvement of fibrosis or NAS versus placebo. ${ }^{41}$ Conflicting results were found in larger studies on biopsy-confirmed NASH patients, where the same dose of sitagliptine $(100 \mathrm{mg})$ given for 1 year improved NAS by ameliorating steatosis and ballooning, regardless of diabetic state, 42 and in another 24-week administration trial showed no superiority compared to placebo in reducing liver fat infiltration in prediabetic patients with NAFLD or those with diabetes mellitus and NAFLD. ${ }^{43}$ Linagliptine, another DDP4 inhibitor, was shown to have both anti-inflammatory and antisteatotic activity in NASH. ${ }^{44}$ It was evaluated in a NASH mouse model as well, in combination with empagliflozine, a sodium-glucose co-transporter (SGLT)-2 inhibitor. A combination of linagliptine and empagliflozine ameliorated NASH with a stronger anti-fibrotic effect. 45 There are still no human studies being conducted 
to verify the safety and utility of linagliptine in the treatment of NASH.

\section{SGLT inhibitors}

SGLT inhibitors act by reducing glucose reabsorption in the proximal tubule in the kidney, leading to glucosuria and plasma glucose reduction. 46 They have been used in treating diabetes mellitus, but as several mouse model studies showed beneficial effect on liver function and prevention of fibrosis associated with $\mathrm{NASH}$, it has become an interesting option for treating NASH in humans. ${ }^{47,48}$ Ipragliflozin, a SGLT2 inhibitor was investigated in a NASH mouse model, where ipragliflozin had a positive effect on free fatty acid serum concentration, liver lipid metabolism, reduced apoptosis and fibrosis. 46 The same results were confirmed in a similar study using a mouse NASH model and 4-week therapy, in which ipragliflozin improved glucose metabolism, reduced insulin resistance, and improved liver steatosis and fibrosis by reducing inflammation and oxidative stress in the liver. ${ }^{48}$ In humans, in patients with diabetes mellitus type 2 and NAFLD, ipragliflozin reduced liver fat (as estimated indirectly by calculating liver fat index). ${ }^{49} \mathrm{~A}$ Japanese study retrospectively included 130 diabetes mellitus patients with proven NASH, and selected patients with altered liver enzymes when adding ipragliflozin to their DPP-4 inhibitor or GLP-1 receptor antagonist therapy, and found after treatment significantly decreased ALT and the Fibrosis-4 score. ${ }^{50}$

In a randomized, active-controlled, open-label trial on 57 patients with diabetes mellitus type 2 and NAFLD who were treated with dapagliflozin ( $5 \mathrm{mg} / \mathrm{d}$ ) for 24 weeks or placebo, hepatic steatosis and stiffness were assessed noninvasively (by transient elastography and controlled attenuation parameter). ${ }^{51}$ Based on their findings, dapagliflozin improved liver steatosis in diabetes mellitus type 2 and NAFLD patients, and ameliorated liver fibrosis only in patients with significant liver fibrosis assessed non-invasively by transient elastography. ${ }^{51}$ In a randomized placebo-controlled double-blind multicenter study on participants with diabetes mellitus type 2 and NAFLD, dapagliflozin monotherapy reduced liver serum markers, cytokeratin (CK) 18-M30 and CK 18-M65, and plasma fibroblast growth factor (FGF)-21.52 A phase 3 study on the histological efficacy and safety of dapagliflozin in NASH patients is ongoing (NCT03723252).

The effect of empagliflozin on liver steatosis in type 2 diabetes mellitus patients and NAFLD was the focus of an investigator-initiated, prospective, open-label, randomized clinical study to examine the effect of $10 \mathrm{mg}$ of empagliflozin per day when included in the treatment of type 2 diabetes mellitus versus standard treatment without empagliflozin. ${ }^{53}$ Hepatic liver fat was measured by magnetic resonance (MR) imaging proton density fat fraction, and found that the empagliflozin group had a significant reduction of liver fat and ALT levels. ${ }^{53}$ A multicenter study on patients with diabetes mellitus type 2 to evaluate the impact of empagliflozin ( 25 $\mathrm{mg}$ daily) or placebo for 24 weeks on lipid content, liver energy metabolism and body composition evaluated by $1 \mathrm{H}$ magnetic resonance (MR) spectroscopy was recently completed, and results are awaited (NCT02637973).

\section{FGF21 analog (pegbelfermin)}

FGF21 is a regulator of energy metabolism and in a study on patients with NAFLD (defined by MR proton spectroscopy) and NASH (defined by biopsy), plasma FGF21 levels were higher in patients with NASH compared to those without NASH or NAFLD. Plasma FGF21 levels correlated positively with the stage of necroinflammation $(p=0.02)$ and fibrosis $(p<0.001)$ but not with steatosis $(p=0.60) .54$ Endogenous FGF2 1 has a short half-life of 1 to 2 hours, but various modification strategies have been used to create longer acting FGF21 analogues. ${ }^{55,56}$ Pegbelfermin is a recombinant analog of human FGF21 that was evaluated in a multicenter randomized, double-blind, placebo-controlled study in biopsyproven NASH in overweight adults (body mass index of $>25$ $\mathrm{kg} / \mathrm{m}^{2}$ ) in subcutaneous two dose group administration (10 $\mathrm{mg}$ daily and $20 \mathrm{mg}$ weekly). ${ }^{56}$ After 16 weeks of treatment, patients in both groups showed a significant amelioration of steatosis (assessed by MR spectroscopy), levels of noninvasive fibrosis biomarker ( $\mathrm{N}$-terminal type III propeptide), and amelioration of liver stiffness and transaminase levels. ${ }^{56} \mathrm{~A}$ phase $2 \mathrm{~b}$ randomized, double-blind, placebo-controlled study evaluating the safety and efficacy of pegblefermin in patients with NASH and stage 3 fibrosis is active but not recruiting any new patients. The primary outcome was determined as achievement of $\geq 1$ stage amelioration in fibrosis without progression of NASH or NASH improvement with no progression of fibrosis (as determined by liver biopsy) (NCT03486899). Results are awaited.

\section{Statins}

Although statins have been extensively used in treating cardiovascular diseases, their use has been widely underestimated in treating NASH, probably due the common misinterpretation that statins damage the liver, as seen in elevation of liver enzymes during treatment. In a multicenter cohort of 1,201 European individuals who underwent liver biopsy for suspected NASH, statin use was recorded in 107 subjects and was associated with an improvement in liver steatosis, NASH and fibrosis stage F2-F4 development; however, this effect was limited in patients with the I148M PNPLA3 variant. 57

\section{Resmetirom (thyroid hormone receptor $\beta$-agonist)}

Resmetirom, a thyroid hormone receptor $\beta$-agonist has been shown in a mouse model to reduce liver steatosis by targeting dyslipidemia. ${ }^{58}$ In a 36 -week randomized, doubleblind, placebo-controlled multicenter study on adults with biopsy-confirmed NASH (fibrosis stages 1-3) and hepatic fat fraction of at least $10 \%$ (assessed by MR proton density fat fraction) resmetirom treatment resulted in improvement in steatosis in NASH patients. ${ }^{59}$ Adverse events were mostly mild or moderate, with a higher incidence of transient diarrhea and nausea caused by the resmetirom. ${ }^{59}$ A phase 3 , multinational study (MAESTRO-NASH) on resmetirom at 80 $\mathrm{mg}$ or $100 \mathrm{mg}$ compared to placebo to achieve NASH resolution on liver histology in non-cirrhotic NASH patients with stage 2 or 3 fibrosis is recruiting and expected to end in 2024 (NCT03900429).

\section{Firsocostat (acetyl-CoA carboxylase inhibitor)}

Firsocostat is an acetyl-CoA carboxylase inhibitor that targets de novo lipogenesis through inhibition of acetyl-CoA to malonyl-CoA conversion. In a phase 2 trial that included 126 patients with NASH and fibrosis, $20 \mathrm{mg}$ daily of firsocostat for 12 weeks showed significant reduction in liver fat by $29 \%$; however, during treatment, an increase in plasma triglyceride levels was recorded, with 16 patients having levels of more than $500 \mathrm{mg} / \mathrm{dL} .{ }^{60}$ Two phase 2 clinical trials on firsocostat in monotherapy or in combination with other therapies for NASH regarding safety, efficacy and tolerabil- 
ity, one on patients with stage 2 and 3 fibrosis and another in participants with bridging fibrosis or compensated cirrhosis are expected to finish soon, and results of these studies are awaited (NCT02781584, NCT03449446).

\section{Drugs modulating hepatocyte injury, inflammation, apoptosis and fibrosis}

Inflammation is a crucial step in NASH pathogenesis, and it has been found that Kupffer cells secrete $\mathrm{C}-\mathrm{C}$ chemokine ligand types 2 (CCL2) in response to hepatocyte injury which, downstream, leads to monocyte recruitment and influx to the liver, where they mature into proinflammatory macrophages. ${ }^{61-63}$ These activated macrophages express proinflammatory cytokines, which in turn activate hepatic stellate cells, promote their survival, and stimulate fibrogenesis. ${ }^{63} \mathrm{C}-\mathrm{C}$ chemokine receptor types 2 (CCR2) and 5 (CCR5), and their ligands, C-C chemokine ligand types 2 (CCL2) and type 5 (CCL5) where found to be up-regulated in NASH. ${ }^{64}$

Cenicriviroc, a dual CCR2/CCR5 antagonist with a long plasma half-life (30-40 $\mathrm{h}$ in humans), was studied in a phase $2 \mathrm{~b}$ study (CENTAUR) in the treatment of NASH and liver fibrosis. ${ }^{63}$ The study evaluated efficacy and safety of $150 \mathrm{mg}$ per day of cenicriviroc over 2 years for obtaining improvement in NAS at the first year relative to screening biopsy, without progression of fibrosis. ${ }^{63}$ After 1 year of cenicriviroc, two times more patients achieved regression of fibrosis without worsening of steatohepatitis compared with placebo $(20 \%$ vs. $10 \% ; p=0.02) .{ }^{63} \mathrm{~A}$ phase III study to evaluate the efficacy and safety of $150 \mathrm{mg}$ per day of cenicriviroc versus placebo for the treatment of liver fibrosis in adult subjects with NASH and stage 2 or 3 liver fibrosis (AURORA trial) is still recruiting patients and results are awaited (NCT03028740).

\section{Farnesoid $X$ receptor agonists}

Farnesoid $X$ receptor (FXR) is a nuclear receptor expressed in the liver, gallbladder, and intestines. It is a modulator of bile acid, glucose and lipid metabolism. ${ }^{65-67}$ In the intestine, FXR modulates FGF15 and FGF19 synthesis and delivery to the liver through the portal circulation. ${ }^{65}$ In the liver, FGF15 and FGF19 stimulate glycogen synthesis and suppress gluconeogenesis but also decrease triglyceride accumulation. ${ }^{66}$ FXR activation reduces activity of SREBP1C, a key transcription factor in regulation of triglyceride synthesis. ${ }^{67}$ Obeticholic acid, which acts as an FXR agonist, has been evaluated in a phase 2 study on NASH patients without cirrhosis (FLINT trial), which randomized 283 patients to $25 \mathrm{mg}$ of obeticholic acid or placebo for 72 weeks. ${ }^{68}$ Although obeticholic acid was successful in the FLINT trial in reducing NAS by 2 points without worsening of fibrosis, a large number of patients developed significant pruritus and experienced a rise in total serum cholesterol and low-density lipoprotein. ${ }^{68}$ Since these changes were attributed to suppression of de novo bile acid synthesis and an escalation in reverse cholesterol transport, future research was focused on development of FXR agonists that would have better efficacy, safety and tolerability profiles. ${ }^{69}$ Tropifexor, a novel FXR agonist, that modulates gene expression in the liver and intestines in low doses with low systemic exposures was found to have a good tolerability profile in healthy volunteers and is now being evaluated in patients with NASH.69 A combination therapy of tropifexor and cenicriviroc is also being evaluated in a phase 2 study (TANDEM trial) in patients with NASH and liver fibrosis (NCT03517540).

\section{Selonsertib (apoptosis signal-regulating kinase 1 in-} hibitor)

Selonsertib is a an apoptosis signal-regulating kinase 1 (known as ASK1) inhibitor that is intended to target the p38/JNK pathway, which is activated by TNFa and intracellular oxidative stress, and results in apoptosis and fibrosis. ${ }^{70} \mathrm{~A}$ phase 2 multicenter study of selonsertib, given for 24 weeks once daily in doses of $6 \mathrm{mg}$ and $18 \mathrm{mg}$, with or without simtuzumab, was undertaken in NASH patients with liver fibrosis (stage 2 or 3 ). ${ }^{71}$ Paired pretreatment and posttreatment liver biopsies and noninvasive diagnostic methods were used to evaluate the efficacy of treatment. After the treatment, a significant number of patients in the $18 \mathrm{mg}$ selonsertib group achieved one or more stage amelioration in fibrosis compared to the $6 \mathrm{mg}$ selonsertibe group and simtuzumab alone ( $43 \%$ vs. $30 \%$ vs. $20 \%$, respectively). ${ }^{71}$ Reduction in fibrosis was associated with reduction in liver stiffness and apoptosis markers measured by noninvasive methods. ${ }^{71}$ However, further trials of selonsertib on NASH patients but with stage 3 fibrosis (STELLAR-3) and stage 4 fibrosis/compensated cirrhosis (STELLAR-4) found that 48 week treatment with selonsertib ( 6 or $18 \mathrm{mg}$ daily dose) had no significant effect on liver serum tests or fibrosis progression evaluated noninvasively. ${ }^{72}$

\section{Drugs targeting gut microbiome changes}

Although the composition of gut microbiome varies among individuals, the prevailing bacterial phyla are Firmicutes and Bacteroidetes, that make up around $90 \%$ of the microbiome, and Actinobacteria and Proteobacteria. The prevailing bacterial phyla is responsible for a specific metabolite profile that can influence liver and overall metabolism, specifically metabolites such as bile acids, lipopolysaccharides and shortchain fatty acids. ${ }^{73}$ It has been shown that the changes in gut microbiome and concentrations of the aforementioned metabolites is important in NAFLD pathogenesis and progression, but a specific microbiome composition in NAFLD has not yet been identified. ${ }^{74,75} \mathrm{~A}$ prospective study on fecal microbiome in adult NAFLD patients, carried out by Loomba et al. ${ }^{76}$ on the association between microbiome composition and advanced stages of fibrosis in NAFLD, identified 37 bacterial species that vary depending on the disease stage. However, the authors state that these results could reflect changes within the microbiome that occur with age and that further studies are needed to see if specific microbial species are responsible for the gut-liver crosstalk and progression of NAFLD. ${ }^{76}$ Prebiotics, probiotics, and antibiotics have been investigated in animal and human studies, in attempts to modify the microbiome and influence NAFLD. In animal studies, it has been shown that prebiotics and synbiotics can $y$ influence gene expression to modify $\beta$-oxidation and lipogenesis, thus effecting liver fat infiltration, inflammation, and insulin resistance. ${ }^{77,78}$ Results in human studies regarding their use in NAFLD were modest; although, most of these studies were not accompanied by a histological confirmation and had a small population sample. ${ }^{79,80} \mathrm{~A}$ recent meta-analysis on the use of prebiotics, probiotics and synbiotics for NAFLD concluded that prebiotic and probiotic use was associated with a reduction in body mass index (BMI) and modest influence on serum aminotransferase levels and lipid profile, without ameliorating inflammation. ${ }^{79}$

\section{Antibiotics}

Antibiotics have been relatively successfully used in treating 
NAFLD. An example is a trial with rifaximin, where a 28-day treatment induced a decrease in BMI, serum aminotransferases, and gamma-glutamyl transferase. ${ }^{80}$ Since then, there have been many trials with opposing results, but the antibiotic treatment for NAFLD and obesity is still intriguing, based on the high number of ongoing trials. ${ }^{81}$ However, although short-term antibiotic treatment could prove beneficial, long-term and frequent use of antibiotics could cause a much greater problem of antibiotic resistance.

\section{Fecal transplantation}

Fecal transplantation, successful in treatment of Clostridium difficile infection, has been a promising treatment for microbial dysbiosis in NAFLD. However, recently published results from a randomized controlled trial on 21 patients with NAFLD that underwent autologous and allogenic fecal transplantation, and were followed up until 6 months from the procedure, found that although allogenic transplantation reduced small intestinal permeability it did not influence insulin resistance (measured by HOMA-IR) or hepatic steatosis measured by MR proton density fat fraction. 82

\section{Modification of bacterial metabolites}

A specific microbiome composition is also responsible for various points in bile acid metabolism and circulation in the body. Enterohepatic circulation of bile acids is regulated by the microbiome since it affects synthesis of amino acids necessary for bile acid liver conjugation as well as the expression of terminal ileum transporters that reabsorb around $95 \%$ of intestinal bile acids. ${ }^{83,84}$ As mentioned in the previous section, the FXR receptor is a nuclear receptor expressed in the liver, gallbladder and intestines, and a modulator of bile acid metabolism. ${ }^{65-67}$ Obeticholic acid is a synthetic derivate of chenodeoxycholic acid that as well as cholic acid is a primary bile acid synthesized in the liver. In study by Friedman et al., 85 obeticholic acid induced suppression of bile acid synthesis (measured by reduced levels of 7a-hydroxy4-cholesten-3-one) and caused an increase in Gram-positive bacteria species ( $S$. thermophilus, L. casei, and L. lactis). The FLINT trial on obeticholic acid in NASH patients, as elaborated earlier, emphasized the need for FXR agonists with better efficacy, safety and tolerability profiles. 68

Specific short chain fatty acids have been found to indirectly influence NASH progression, as well as previously described bile acids, in interplay with microbiome composition. ${ }^{86}$ Short chain fatty acids are products of complex carbohydrate fermentation that cannot be digested by the organism, and the most prevalent are acetate, butyrate, and propionate. ${ }^{87}$ They exert their effects by binding to specific receptors in the colon to increase GLP-1 and other insulin sensitizing peptides. ${ }^{88}$ Their overall effects on liver, skeletal muscle and adipose tissue in vitro promote de novo lipid synthesis, fat oxidation, anabolism, and insulin sensitivity. 89 Moreover, studies have shown that they strengthen the intestinal barrier and reduce gut permeability and, by that, exhibit anti-inflammatory effects that are an important component in NASH development. ${ }^{90,91}$ In humans, the interplay between specific short fatty acids and the residing microbiome is more complex, and studies that addressed specific therapeutic procedures influencing short fatty acid intestine composition are inconsistent in their conclusions, so further research is necessary. ${ }^{89}$

Lipopolysaccharides are a structural component of Gramnegative bacteria and of endotoxins that in healthy microbiota and intact intestinal barrier enter the hepatic circulation in only small amount and are thereby eliminated by Kupffer cells after recognition by Toll-like receptors. ${ }^{92}$ In patients with $\mathrm{NASH}$, there is an up-regulation of the pro-inflammatory response in liver that results from a major influx of lipopolysaccharides and other bacteria metabolites due to impaired intestinal barrier and altered microbiome. ${ }^{93}$ Agents that could affect this pathway are extensively researched, and a recently published study on sevelamer (that acts as a hydrophilic bile acid sequestrant) showed great potential in affecting liver fibrosis in a diet-induced NASH animal model. ${ }^{94}$ Sevelamer improved the composition of the gut microbiome, improved the intestinal barrier, promoted fecal excretion of lipopolysaccharides and, by that, reduced the concentration of lipopolysaccharides in liver and suppressed the proinflammatory Toll-like receptor pathway. ${ }^{94}$ Studies on sevelamer in human NASH patients have not yet been conducted.

\section{Conclusions}

NASH represents an important global health burden with significant morbidity and the available treatment options are still unsatisfactory. However, new treatments for NASH are emerging. There is a large number of new drugs that are being tested in the preclinical setting and understanding NASH pathogenesis has a crucial role in their development. The microbiome composition has been shown to be related to changes in gut permeability, leaking lipopolysaccharides, and metabolism of short-chain fatty acids, all indirectly influencing proinflammatory and profibrotic pathways in the liver. Fecal microbiota transplantation and influencing the microbiome composition through bile acids and agents affecting other microbial metabolites have, in that way, been recognized as possible mechanisms to influence the development of NASH, potentially to reverse the changes preceding NASH and even influence NASH stage regression.

After years of not being able to actively treat NASH other than with diet and exercise modification, with only limited pharmacological possibilities, we are now expecting drugs which target specific points in NASH pathogenesis. Although $\mathrm{NASH}$ is a multifactorial disease, in the future, we could identify the predominating pathological mechanism and, by choosing specific medications, tailor the treatment for every patient individually.

\section{Funding}

None to declare.

\section{Conflict of interest}

The authors have no conflict of interests related to this publication.

\section{Author contributions}

Study concept and design (MD, SSS), acquisition of data (SSS, LVJ, DH), analysis and interpretation of data (MD, SSS, LVJ, DH), drafting of the manuscript (SSS, LVJ), critical revision of the manuscript for important intellectual content (MD, LVJ, DH), administrative, technical, or material support, study supervision (LSD, MD).

\section{References}

[1] Chalasani N, Younossi Z, Lavine JE, Charlton M, Cusi K, Rinella M, et al. 
The diagnosis and management of nonalcoholic fatty liver disease: Practice guidance from the American Association for the Study of Liver Diseases. Hepatology 2018;67:328-357. doi:10.1002/hep.29367.

[2] Duseja A, Singh SP, Saraswat VA, Acharya SK, Chawla YK, Chowdhury S, et al. Non-alcoholic fatty liver disease and metabolic syndrome-position paper of the Indian National Association for the Study of the Liver, Endocrine Society of India, Indian College of Cardiology and Indian Society of Gastroenterology. J Clin Exp Hepatol 2015;5:51-68. doi:10.1016/j. jceh.2015.02.006.

[3] Estes C, Razavi H, Loomba R, Younossi Z, Sanyal AJ. Modeling the epidemic of nonalcoholic fatty liver disease demonstrates an exponential increase in burden of disease. Hepatology 2018;67:123-133. doi:10.1002/ hep. 29466.

[4] Estes C, Anstee QM, Arias-Loste MT, Bantel H, Bellentani S, Caballeria J, et al. Modeling NAFLD disease burden in China, France, Germany, Italy, Japan, Spain, United Kingdom, and United States for the period 2016-2030. J Hepatol 2018;69:896-904. doi:10.1016/j.jhep.2018.05.036.

[5] Younossi ZM, Otgonsuren M, Henry L, Venkatesan C, Mishra A, Erario M, et al. Association of nonalcoholic fatty liver disease (NAFLD) with hepatocellular carcinoma (HCC) in the United States from 2004 to 2009. Hepatology 2015;62:1723-1730. doi:10.1002/hep.28123.

[6] Younossi ZM, Koenig AB, Abdelatif D, Fazel Y, Henry L, Wymer M. Global epidemiology of nonalcoholic fatty liver disease-Meta-analytic assessment of prevalence, incidence, and outcomes. Hepatology 2016;64:73-84. doi:10.1002/hep.28431

[7] Matteoni CA, Younossi ZM, Gramlich T, Boparai N, Liu YC, McCullough AJ. Nonalcoholic fatty liver disease: a spectrum of clinical and pathological severity. Gastroenterology 1999;116:1413-1419. doi:10.1016/s00165085(99)70506-8.

[8] Sheka AC, Adeyi O, Thompson J, Hameed B, Crawford PA, Ikramuddin S. Nonalcoholic steatohepatitis: A review. JAMA 2020:323:1175-1183. doi:10.1001/jama.2020.2298.

[9] van der Windt DJ, Sud V, Zhang H, Tsung A, Huang $H$. The effects of physical exercise on fatty liver disease. Gene Expr 2018;18:89-101. doi:10.37 27/105221617X15124844266408

[10] Musso G, Cassader M, Rosina F, Gambino R. Impact of current treatments on liver disease, glucose metabolism and cardiovascular risk in non-alcoholic fatty liver disease (NAFLD): a systematic review and meta-analysis of randomised trials. Diabetologia 2012;55:885-904. doi:10.1007/s00125011-2446-4.

[11] EASL-EASD-EASO Clinical Practice Guidelines for the management of nonalcoholic fatty liver disease. J Hepatol 2016;64:1388-1402. doi:10.1016/j. jhep.2015.11.004

[12] Sanyal AJ, Chalasani N, Kowdley KV, McCullough A, Diehl AM, Bass NM, et al. Pioglitazone, vitamin $\mathrm{E}$, or placebo for nonalcoholic steatohepatitis. N Engl J Med 2010;362:1675-1685. doi:10.1056/NEJMoa0907929.

[13] Gerss ], Köpcke W. The questionable association of vitamin E supplementation and mortality-inconsistent results of different meta-analytic approaches. Cell Mol Biol (Noisy-le-grand) 2009;55 Suppl:OL1111-OL1120.

[14] Eslam M, Sanyal AJ, George J. MAFLD: A consensus-driven proposed nomenclature for metabolic associated fatty liver disease. Gastroenterology menclature for metabolic associated fatty liver disease. Gast

[15] Muthiah MD, Sanyal AJ. Current management of non-alcoholic steatohepatitis. Liver Int 2020;40 Suppl 1:89-95. doi:10.1111/liv.14355.

[16] Grygiel-Górniak B. Peroxisome proliferator-activated receptors and their ligands: nutritional and clinical implications-a review. Nutr J 2014;13:17 doi:10.1186/1475-2891-13-17.

[17] Pawlak M, Lefebvre P, Staels B. Molecular mechanism of PPARa action and its impact on lipid metabolism, inflammation and fibrosis in nonalcoholic fatty liver disease. J Hepatol 2015;62:720-733. doi:10.1016/j. jhep.2014.10.039.

[18] Lefere S, Puengel T, Hundertmark J, Penners C, Frank AK, Guillot A, et al Differential effects of selective- and pan-PPAR agonists on experimental steatohepatitis and hepatic macrophages. J Hepatol 2020;73:757-770. steatohepatitis and hepatic macror
doi:10.1016/j.jhep.2020.04.025.

[19] Fuchs CD, Traussnigg SA, Trauner M. Nuclear receptor modulation for the treatment of nonalcoholic fatty liver disease. Semin Liver Dis 2016;36:6986. doi:10.1055/s-0036-1571296.

[20] Maeda N, Takahashi M, Funahashi T, Kihara S, Nishizawa H, Kishida K, et al. PPARgamma ligands increase expression and plasma concentrations of adiponectin, an adipose-derived protein. Diabetes 2001;50:2094-2099. doi:10.2337/diabetes.50.9.2094.

[21] Bojic LA, Huff MW. Peroxisome proliferator-activated receptor $\delta$ : a multifaceted metabolic player. Curr Opin Lipidol 2013;24:171-177. doi:10.1097/ eted metabolic player. Curr

[22] Odegaard JI, Ricardo-Gonzalez RR, Red Eagle A, Vats D, Morel CR, Goforth $\mathrm{MH}$, et al. Alternative M2 activation of Kupffer cells by PPARdelta ameliorates obesity-induced insulin resistance. Cell Metab 2008;7:496-507. doi:10.1016/j.cmet.2008.04.003.

[23] Ratziu V, Harrison SA, Francque S, Bedossa P, Lehert P, Serfaty L, et al. Elafibranor, an agonist of the peroxisome proliferator-activated receptor-a and $-\delta$, induces resolution of nonalcoholic steatohepatitis without fibrosis worsening. Gastroenterology 2016;150:1147-1159.e5. doi:10.1053/j. gis worsening. Gastroer

[24] Sumida Y, Okanoue T, Nakajima A. Phase 3 drug pipelines in the treatment of non-alcoholic steatohepatitis. Hepatol Res 2019;49:1256-1262. doi:10.1111/hepr.13425.

[25] Krishna AV, Sah RK, Ali M. Saroglitazar: a novel dual acting peroxisome proliferator activated receptor (PPAR) in dyslipidemia associated with T2DM. Ejpmr 2017;4:680-684.

[26] Kaul U, Parmar D, Manjunath K, Shah M, Parmar K, Patil KP, et al. New dual peroxisome proliferator activated receptor agonist-Saroglitazar in diabetic dyslipidemia and non-alcoholic fatty liver disease: integrated analysis of the real world evidence. Cardiovasc Diabetol 2019;18:80. doi:10.1186/ s12933-019-0884-3.

[27] Boeckmans J, Natale A, Rombaut M, Buyl K, Rogiers V, De Kock J, et al. Anti-NASH drug development hitches a lift on PPAR agonism. Cells 2019;9:37. doi:10.3390/cells9010037.

[28] Sumida Y, Yoneda M, Tokushige K, Kawanaka M, Fujii H, Yoneda M, et al. Antidiabetic therapy in the treatment of nonalcoholic steatohepatitis. Int J Mol Sci 2020;21:1907. doi:10.3390/ijms21061907.

[29] Gupta NA, Mells J, Dunham RM, Grakoui A, Handy J, Saxena NK, et al. Glucagon-like peptide-1 receptor is present on human hepatocytes and has a direct role in decreasing hepatic steatosis in vitro by modulating elements of the insulin signaling pathway. Hepatology 2010;51:1584-1592. doi: 10.1002 /hep. 23569 .

[30] de Mesquita FC, Guixé-Muntet S, Fernández-Iglesias A, Maeso-Díaz R, Vila $S$, Hide $D$, et al. Liraglutide improves liver microvascular dysfunction in cirrhosis: Evidence from translational studies. Sci Rep 2017:7:3255. doi:10.1038/s41598-017-02866-y.

[31] Tølbøl KS, Kristiansen MN, Hansen $\mathrm{HH}$, Veidal SS, Rigbolt KT, Gillum MP et al. Metabolic and hepatic effects of liraglutide, obeticholic acid and elafibranor in diet-induced obese mouse models of biopsy-confirmed elafibranor in diet-induced obese mouse models of biopsy-confirmed nonalcoholic steatohepatitis.
doi:10.3748/wjg.v24.i2.179.

[32] Armstrong MJ, Gaunt P, Aithal GP, Barton D, Hull D, Parker R, et al. Liraglutide safety and efficacy in patients with non-alcoholic steatohepatitis (LEAN): a multicentre, double-blind, randomised, placebo-controlled phase 2 study. Lancet 2016;387:679-690. doi:10.1016/S0140-6736(15)00803$\mathrm{X}$.

[33] Khoo J, Hsiang JC, Taneja R, Koo SH, Soon GH, Kam CJ, et al. Randomized trial comparing effects of weight loss by liraglutide with lifestyle modification in non-alcoholic fatty liver disease. Liver Int 2019:39:941-949. doi:10.1111/liv.14065

[34] Kalavalapalli S, Bril F, Guingab J, Vergara A, Garrett TJ, Sunny NE, et al. Impact of exenatide on mitochondrial lipid metabolism in mice with nonalcoholic steatohepatitis. J Endocrinol 2019;241:293-305. doi:10.1530/ JOE-19-0007.

[35] Shao N, Kuang HY, Hao M, Gao XY, Lin WJ, Zou W. Benefits of exenatide on obesity and non-alcoholic fatty liver disease with elevated liver enzymes in patients with type 2 diabetes. Diabetes Metab Res Rev 2014;30:521-529. doi:10.1002/dmrr.2561.

[36] Kenny PR, Brady DE, Torres DM, Ragozzino L, Chalasani N, Harrison SA. Exenatide in the treatment of diabetic patients with non-alcoholic steatohepatitis: a case series. Am J Gastroenterol 2010;105:2707-2709. tohepatitis: a case series.
doi: $10.1038 /$ ajg. 2010.363 .

[37] Marso SP, Holst AG, Vilsbøll T. Semaglutide and cardiovascular outcomes in patients with type 2 diabetes. N Engl J Med 2017;376:891-892. doi:10.1056/NEJMc1615712.

[38] Newsome P, Francque S, Harrison S, Ratziu V, Van Gaal L, Calanna S, et al. Effect of semaglutide on liver enzymes and markers of inflammation in subjects with type 2 diabetes and/or obesity. Aliment Pharmacol Ther 2019;50:193-203. doi:10.1111/apt.15316.

[39] Shirakawa J, Fujii H, Ohnuma K, Sato K, Ito Y, Kaji M, et al. Diet-induced adipose tissue inflammation and liver steatosis are prevented by DPP-4 inhibition in diabetic mice. Diabetes 2011;60:1246-1257. doi:10.2337/ db10-1338.

[40] Kawaguchi T, Nakano D, Koga H, Torimura T. Effects of a DPP4 inhibitor on progression of nash-related HCC and the p62/ Keap1/Nrf2-pentose phosphate pathway in a mouse model. Liver Cancer 2019;8:359-372. doi: $10.1159 / 000491763$.

[41] Joy TR, McKenzie CA, Tirona RG, Summers K, Seney S, Chakrabarti S, et al. Sitagliptin in patients with non-alcoholic steatohepatitis: A randomized, placebo-controlled trial. World J Gastroenterol 2017;23:141-150. doi:10.3748/wjg.v23.i1.141.

[42] Alam S, Ghosh J, Mustafa G, Kamal M, Ahmad N. Effect of sitagliptin on hepatic histological activity and fibrosis of nonalcoholic steatohepatitis patients: a 1-year randomized control trial. Hepat Med 2018;10:23-31. doi: 10.2147/HMER.S158053

[43] Cui J, Philo L, Nguyen P, Hofflich H, Hernandez C, Bettencourt R, et al. Sitagliptin vs. placebo for non-alcoholic fatty liver disease: A randomized controlled trial. J Hepatol 2016;65:369-376. doi:10.1016/j.jhep.2016.04.021.

[44] Klein T, Fujii M, Sandel J, Shibazaki Y, Wakamatsu K, Mark M, et al. Linagliptin alleviates hepatic steatosis and inflammation in a mouse model of non-alcoholic steatohepatitis. Med Mol Morphol 2014;47:137-149. doi:10.1007/s00795-013-0053-9.

[45] Jojima T, Tomotsune T, Iijima T, Akimoto K, Suzuki K, Aso Y. Empagliflozin (an SGLT2 inhibitor), alone or in combination with linagliptin (a DPP-4 inhibitor), prevents steatohepatitis in a novel mouse model of nonalcoholic steatohepatitis and diabetes. Diabetol Metab Syndr 2016;8:45. doi:10.1186/s13098-016-0169-x.

[46] Tahara A, Kurosaki E, Yokono M, Yamajuku D, Kihara R, Hayashizaki Y, et al. Effects of SGLT2 selective inhibitor ipragliflozin on hyperglycemia, hyperlipidemia, hepatic steatosis, oxidative stress, inflammation, and obesity in type 2 diabetic mice. Eur J Pharmacol 2013;715:246-255. doi:10.1016/j. ejphar.2013.05.014.

[47] Honda $Y$, Imajo $K$, Kato T, Kessoku T, Ogawa $Y$, Tomeno W, et al. The selective SGLT2 inhibitor ipragliflozin has a therapeutic effect on nonalcoholic steatohepatitis in mice. PLoS One 2016;11:e0146337. doi:10.1371/journal.pone.0146337.

[48] Tahara A, Takasu T. SGLT2 inhibitor ipragliflozin alone and combined with pioglitazone prevents progression of nonalcoholic steatohepatitis in a type 2 diabetes rodent model. Physiol Rep 2019;7:e14286. doi:10.14814/ phy2.14286. 
[49] Takase T, Nakamura A, Miyoshi H, Yamamoto C, Atsumi T. Amelioration of fatty liver index in patients with type 2 diabetes on ipragliflozin: an association with glucose-lowering effects. Endocr J 2017;64:363-367. doi:10.1507/endocrj.EJ16-0295.

[50] Ohki T, Isogawa A, Toda N, Tagawa K. Effectiveness of ipragliflozin, a sodium-glucose co-transporter 2 inhibitor, as a second-line treatment for non-alcoholic fatty liver disease patients with type 2 diabetes mellitus who do not respond to incretin-based therapies including glucagon-like peptide-1 analogs and dipeptidyl peptidase-4 inhibitors. Clin Drug Investig 2016;36:313-319. doi:10.1007/s40261-016-0383-1.

[51] Shimizu M, Suzuki K, Kato K, Jojima T, Iijima T, Murohisa T, et al. Evaluation of the effects of dapagliflozin, a sodium-glucose co-transporter-2 inhibitor, on hepatic steatosis and fibrosis using transient elastography in patients with type 2 diabetes and non-alcoholic fatty liver disease. Diabetes Obes Metab 2019;21:285-292. doi:10.1111/dom.13520.

[52] Eriksson JW, Lundkvist P, Jansson PA, Johansson L, Kvarnström M, Moris L, et al. Effects of dapagliflozin and n-3 carboxylic acids on non-alcoholic fatty liver disease in people with type 2 diabetes: a double-blind randomised placebo-controlled study. Diabetologia 2018;61:1923-1934. doi:10.1007/ placebo-controlled study
s00125-018-4675-2.

[53] Kuchay MS, Krishan S, Mishra SK, Farooqui KJ, Singh MK, Wasir JS, et al. Effect of empagliflozin on liver fat in patients with type 2 diabetes and nonalcoholic fatty liver disease: a randomized controlled trial (E-LIFT Trial). Diabetes Care 2018;41:1801-1808. doi:10.2337/dc18-0165.

[54] Barb D, Bril F, Kalavalapalli S, Cusi K. Plasma fibroblast growth factor 21 is associated with severity of nonalcoholic steatohepatitis in patients with obesity and type 2 diabetes. J Clin Endocrinol Metab 2019;104:33273336. doi:10.1210/jc.2018-02414.

[55] Sonoda J, Chen MZ, Baruch A. FGF21-receptor agonists: an emerging therapeutic class for obesity-related diseases. Horm Mol Biol Clin Investig 2017;30:20070002. doi:10.1515/hmbci-2017-0002.

[56] Sanyal A, Charles ED, Neuschwander-Tetri BA, Loomba R, Harrison SA Abdelmalek MF, et al. Pegbelfermin (BMS-986036), a PEGylated fibroblast growth factor 21 analogue, in patients with non-alcoholic steatohepatitis: a randomised, double-blind, placebo-controlled, phase 2 a trial. Lancet 2019;392:2705-2717. doi:10.1016/S0140-6736(18)31785-9.

[57] Dongiovanni P, Petta S, Mannisto V, Mancina RM, Pipitone R, Karja V, et al. Statin use and non-alcoholic steatohepatitis in at risk individuals. J Hepatol 2015;63:705-712. doi:10.1016/j.jhep.2015.05.006.

[58] Vatner DF, Weismann D, Beddow SA, Kumashiro N, Erion DM, Liao XH, et al. Thyroid hormone receptor- $\beta$ agonists prevent hepatic steatosis in fatfed rats but impair insulin sensitivity via discrete pathways. Am J Physio Endocrinol Metab 2013;305:E89-E100. doi:10.1152/ajpendo.00573.2012.

[59] Harrison SA, Bashir MR, Guy CD, Zhou R, Moylan CA, Frias JP, et al. Resmetirom (MGL-3196) for the treatment of non-alcoholic steatohepatitis: a multicentre, randomised, double-blind, placebo-controlled, phase 2 trial.
Lancet 2019;394:2012-2024. doi:10.1016/S0140-6736(19)32517-6.

[60] Alkhouri N, Lawitz E, Noureddin M, DeFronzo R, Shulman GI. GS-0976 (Firsocostat): an investigational liver-directed acetyl-CoA carboxylase (ACC) inhibitor for the treatment of non-alcoholic steatohepatitis (NASH). Expert Opin Investig Drugs 2020;29:135-141. doi:10.1080/13543784.2020.166 8374.

[61] Miura K, Yang L, van Rooijen N, Ohnishi H, Seki E. Hepatic recruitment of macrophages promotes nonalcoholic steatohepatitis through CCR2. Am J Physiol Gastrointest Liver Physiol 2012;302:G1310-G1321. doi:10.1152/ ajpgi.00365.2011.

[62] Pradere JP, Kluwe J, De Minicis S, Jiao JJ, Gwak GY, Dapito DH, et al. Hepatic macrophages but not dendritic cells contribute to liver fibrosis by promoting the survival of activated hepatic stellate cells in mice. Hepatology 2013;58:1461-1473. doi:10.1002/hep.26429.

[63] Friedman S, Sanyal A, Goodman Z, Lefebvre E, Gottwald M, Fischer L, et al. Efficacy and safety study of cenicriviroc for the treatment of non-alcoholic steatohepatitis in adult subjects with liver fibrosis: CENTAUR Phase 2b study design. Contemp Clin Trials 2016;47:356-365. doi:10.1016/j. cct.2016.02.012.

[64] Bertola A, Bonnafous S, Anty R, Patouraux S, Saint-Paul MC, Iannelli A, et al. Hepatic expression patterns of inflammatory and immune response genes associated with obesity and NASH in morbidly obese patients. PLoS One 2010;5:e13577. doi:10.1371/journal.pone.0013577.

[65] Kir S, Beddow SA, Samuel VT, Miller P, Previs SF, Suino-Powell K, et al. FGF19 as a postprandial, insulin-independent activator of hepatic protein and glycogen synthesis. Science 2011;331:1621-1624. doi:10.1126/science.1198363.

[66] Alvarez-Sola G, Uriarte I, Latasa MU, Fernandez-Barrena MG, Urtasun R, Elizalde $M$, et al. Fibroblast growth factor $15 / 19$ (FGF15/19) protects from diet-induced hepatic steatosis: development of an FGF19-based chimeric molecule to promote fatty liver regeneration. Gut 2017;66:1818-1828. doi:10.1136/gutjnl-2016-312975.

[67] Chiang JY. Bile acids: regulation of synthesis. J Lipid Res 2009;50:19551966. doi:10.1194/jlr.R900010-JLR200.

[68] Neuschwander-Tetri BA, Loomba R, Sanyal AJ, Lavine JE, Van Natta ML, Abdelmalek MF, et al. Farnesoid $\mathrm{X}$ nuclear receptor ligand obeticholic acid for non-cirrhotic, non-alcoholic steatohepatitis (FLINT): a multicentre, randomised, placebo-controlled trial. Lancet 2015;385:956-965. tre, randomised, placebo-controlled
doi:10.1016/S0140-6736(14)61933-4.

[69] Tully DC, Rucker PV, Chianelli D, Williams J, Vidal A, Alper PB, et al. Discovery of tropifexor (LJN452), a highly potent non-bile acid FXR agonist for the treatment of cholestatic liver diseases and nonalcoholic steatohepatitis (NASH). J Med Chem 2017;60:9960-9973. doi:10.1021/acs. jmedchem.7b00907.
[70] Brenner C, Galluzzi L, Kepp O, Kroemer G. Decoding cell death signals in liver inflammation. J Hepatol 2013;59:583-594. doi:10.1016/j. jhep.2013.03.033.

[71] Loomba R, Lawitz E, Mantry PS, Jayakumar S, Caldwell SH, Arnold H, et al. The ASK1 inhibitor selonsertib in patients with nonalcoholic steatohepatitis: A randomized, phase 2 trial. Hepatology 2018;67:549-559. doi:10.1002/hep.29514.

[72] Harrison SA, Wong VW, Okanoue T, Bzowej N, Vuppalanchi R, Younes Z, et al. Selonsertib for patients with bridging fibrosis or compensated cirrhosis due to NASH: Results from randomized phase III STELLAR trials. J Hepatol 2020;73:26-39. doi:10.1016/j.jhep.2020.02.027.

[73] Liu Q, Liu S, Chen L, Zhao Z, Du S, Dong Q, et al. Role and effective therapeutic target of gut microbiota in NAFLD/NASH. Exp Ther Med 2019;18:1935-1944. doi:10.3892/etm.2019.7781.

[74] Clemente MG, Mandato C, Poeta M, Vajro P. Pediatric non-alcoholic fatty liver disease: Recent solutions, unresolved issues, and future research directions. World J Gastroenterol 2016;22:8078-8093. doi:10.3748/wjg. v22.i36.8078.

[75] Wieland A, Frank DN, Harnke B, Bambha K. Systematic review: microbial dysbiosis and nonalcoholic fatty liver disease. Aliment Pharmacol Ther 2015;42:1051-1063. doi:10.1111/apt.13376

[76] Loomba R, Seguritan V, Li W, Long T, Klitgord N, Bhatt A, et al. Gut microbiome-based metagenomic signature for non-invasive detection of advanced fibrosis in human nonalcoholic fatty liver disease. Cell Metab 2017;25:1054-1062.e5. doi:10.1016/j.cmet.2017.04.001.

[77] Dos Santos Aguiar L, Reis MB, Guanabara CC, Júnior OA, Ribeiro DA, Sala P. Prebiotic and synbiotic modifications of beta oxidation and lipogenic gene expression after experimental hypercholesterolemia in rat liver. Front Microbiol 2017;8:2010. doi:10.3389/fmicb.2017.02010.

[78] Xu RY, Wan YP, Fang QY, Lu W, Cai W. Supplementation with probiotics modifies gut flora and attenuates liver fat accumulation in rat nonalcoholic fatty liver disease model. J Clin Biochem Nutr 2012;50:72-77. doi: $10.3164 /$ jcbn.11-38.

[79] Loman BR, Hernández-Saavedra D, An R, Rector RS. Prebiotic and probiotic treatment of nonalcoholic fatty liver disease: a systematic review and meta-analysis. Nutr Rev 2018;76:822-839. doi:10.1093/nutrit/nuy031.

[80] Gangarapu V, Ince AT, Baysal B, Kayar Y, Kılıç U, Gök Ö, et al. Efficacy of rifaximin on circulating endotoxins and cytokines in patients with nonalcoholic fatty liver disease. Eur J Gastroenterol Hepatol 2015;27:840-845. doi:10.1097/MEG.0000000000000348.

[81] Chen HT, Huang HL, Li YQ, Xu HM, Zhou YJ. Therapeutic advances in nonalcoholic fatty liver disease: A microbiota-centered view. World J Gastroenterol 2020;26:1901-1911. doi:10.3748/wjg.v26.i16.1901.

[82] Craven L, Rahman A, Nair Parvathy S, Beaton M, Silverman J, Oumosani $\mathrm{K}$, et al. Allogenic fecal microbiota transplantation in patients with nonalcoholic fatty liver disease improves abnormal small intestinal permeability: A randomized control trial. Am J Gastroenterol 2020;115:1055-1065. doi: $10.14309 /$ ajg.0000000000000661.

[83] Mouzaki M, Loomba R. Insights into the evolving role of the gut microbiome in nonalcoholic fatty liver disease: rationale and prospects for therapeutic intervention. Therap Adv Gastroenterol 2019;12:1756284819858470. doi:10.1177/1756284819858470

[84] Wahlström A, Sayin SI, Marschall HU, Bäckhed F. Intestinal crosstalk between bile acids and microbiota and its impact on host metabolism. Cell Metab 2016;24:41-50. doi:10.1016/j.cmet.2016.05.005.

[85] Friedman ES, Li Y, Shen TD, Jiang J, Chau L, Adorini L, et al. FXR-dependent modulation of the human small intestinal microbiome by the bile acid derivative obeticholic acid. Gastroenterology 2018;155:1741-1752.e5. doi:10.1053/j.gastro.2018.08.022

[86] Zhou D, Chen YW, Zhao ZH, Yang RX, Xin FZ, Liu XL, et al. Sodium butyrate reduces high-fat diet-induced non-alcoholic steatohepatitis through upregulation of hepatic GLP-1R expression. Exp Mol Med 2018;50:1-12. doi:10.1038/s12276-018-0183-1.

[87] Canfora EE, Meex RCR, Venema K, Blaak EE. Gut microbial metabolites in obesity, NAFLD and T2DM. Nat Rev Endocrinol 2019;15:261-273. in obesity, NAFLD and T2DM.

[88] Gao Z, Yin J, Zhang J, Ward RE, Martin RJ, Lefevre M, et al. Butyrate improves insulin sensitivity and increases energy expenditure in mice. Diabetes 2009;58:1509-1517. doi:10.2337/db08-1637.

[89] Canfora EE, Jocken JW, Blaak EE. Short-chain fatty acids in control of body weight and insulin sensitivity. Nat Rev Endocrinol 2015;11:577-591. doi:10.1038/nrendo.2015.128.

[90] Suzuki T, Yoshida S, Hara H. Physiological concentrations of short-chain fatty acids immediately suppress colonic epithelial permeability. $\mathrm{Br}$ ] Nutr fatty acids immediately suppress colonic epithelial perm

[91] Elamin EE, Masclee AA, Dekker J, Pieters HJ, Jonkers DM. Short-chain fatty acids activate AMP-activated protein kinase and ameliorate ethanolinduced intestinal barrier dysfunction in Caco-2 cell monolayers. J Nutr 2013;143:1872-1881. doi:10.3945/jn.113.179549.

[92] Roy S, Benz F, Luedde T, Roderburg C. The role of miRNAs in the regulation of inflammatory processes during hepatofibrogenesis. Hepatobiliary Surg Nutr 2015;4:24-33. doi:10.3978/j.issn.2304-3881.2015.01.05.

[93] Liu J, Zhuang ZJ, Bian DX, Ma XJ, Xun YH, Yang WJ, et al. Toll-like receptor-4 signalling in the progression of non-alcoholic fatty liver disease induced by high-fat and high-fructose diet in mice. Clin Exp Pharmacol Physiol 2014;41:482-488. doi:10.1111/1440-1681.12241.

[94] Tsuji Y, Kaji K, Kitade M, Kaya D, Kitagawa K, Ozutsumi T, et al. Bile acid sequestrant, sevelamer ameliorates hepatic fibrosis with reduced overload of endogenous lipopolysaccharide in experimental nonalcoholic steatohepatitis. Microorganisms 2020;8:925. doi:10.3390/microorganisms8060925. 